\title{
Chunk-based resource allocation in multi-cell OFDMA systems with fairness guarantee
}

\author{
Mahmoud M. Selim ${ }^{1,3 a)}$, Osamu Muta ${ }^{2}$, Hossam M. H. Shalaby ${ }^{1}$, \\ and Hiroshi Furukawa ${ }^{3}$ \\ ${ }^{1}$ Egypt-Japan University of Science and Technology (E-JUST), \\ New Borg Al-Arab, Alexandria, Egypt \\ ${ }^{2}$ Center for Japan-Egypt Cooperation in Science and Technology, \\ Kyushu University, Fukuoka-shi, Fukuoka, Japan \\ ${ }^{3}$ Graduate School of Information Science and Electrical Engineering, \\ Kyushu University \\ a)mahmoud.sleem@ejust.edu.eg
}

\begin{abstract}
Resource allocation (RA) in multi-cell OFDMA systems is very important for maximizing system throughput. Although sub-channel RA is optimal in terms of system throughput, more interest is given to chunk-based RA to simplify RA algorithms and minimize required signalling. In this paper, we propose a fairness-aware chunk-based RA algorithm for the downlink transmission of multi-cell OFDMA system with fractional frequency reuse (FFR) adoption. Simulation results reveal that our proposed algorithm outperforms two reference algorithms in the literature in terms of some system metrics such as average spectral efficiency (SE), users' fairness and cell-edge users' rates.
\end{abstract}

Keywords: resource allocation, multi-cell, fairness, FFR, chunk, OFDMA Classification: Wireless Communication Technologies

\section{References}

[1] 3GPP, Requirements for further advancements for E-UTRA (LTE-Advanced), 3rd Generation Partnership Project (3GPP), TR 36.913, Jun. 2008.

[2] R1-050507, Soft frequency reuse scheme for UTRAN LTE, Tech. Rep. 3GPP TSG-RAN WG1 Meeting 41, May 2005.

[3] M. M. Selim, S. Elnoubi, H. Shalaby, O. Muta, and H. Furukawa, "Reduced complexity QoS aware resource allocation technique for MISO-OFDMA systems," 2014 International Conference on Computing, Networking and Communications (ICNC), pp. 950-954, 2014. DOI:10.1109/ICCNC.2014. 6785465

[4] M. M. Selim, O. Muta, H. M. Shalaby, and H. Furukawa, "Low complexity QoS-aware subcarrier allocation for MISO-OFDMA systems," IEICE Commun. Express, vol. 3, no. 8, pp. 235-240, 2014. DOI:10.1587/comex.3.235

[5] H. Zhu and J. Wang, "Frequency reuse in chunk-based multi-cell OFDMA systems," 2012 IEEE International Conference on Communications (ICC), pp. 4668-4672, 2012. DOI:10.1109/ICC.2012.6364442 
[6] S. H. Ali and V. C. Leung, "Dynamic frequency allocation in fractional frequency reused OFDMA networks," IEEE Trans. Wireless Commun., vol. 8, no. 8, pp. 4286-4295, 2009. DOI:10.1109/TWC.2009.081146

[7] A. J. Goldsmith and S.-G. Chua, "Variable-rate variable-power M-QAM for fading channels," IEEE Trans. Commun., vol. 45, no. 10, pp. 1218-1230, 1997. DOI:10.1109/26.634685

[8] R. Jain, D.-M. Chiu, and W. R. Hawe, A Quantitative Measure of Fairness and Discrimination for Resource Allocation in Shared Computer System, Eastern Research Laboratory, Digital Equipment Corporation, 1984.

\section{Introduction}

Orthogonal frequency division multiple access (OFDMA) is exploited in modern wireless systems to permit wide-band data services [1]. Moreover, fractional frequency reuse (FFR) [2] is known for solving the co-channel interference problem encountered by cell-edge users in multi-cell universal frequency reuse systems. Thus, FFR divides the macro-cell coverage area into center-region with universal frequency reuse and edge-region with reuse factor less than one to minimize number of interferers.

Many algorithms have been previously proposed to solve resource allocation (RA) problem on a sub-channel basis [3, 4]. However, RA on a sub-channel basis has two main drawbacks; higher complexity and large amount of channel information to be fed-back to the base-station (BS). Therefore, it is desirable to group a number of contiguous sub-channels into one chunk and RA is done on a chunk basis rather than sub-channel basis.

The main contribution of this paper is proposing a fairness-aware chunk-based RA algorithm for the downlink transmission of multi-user multi-cell OFDMA system with FFR adoption. We compare our proposed RA algorithm with two different algorithms. The first one [5] is a maximizing capacity two-step RA algorithm that firstly allocates different chunks among users based on only small scale fading channel conditions $^{1}$, unlike our proposed algorithm that considers users geographic location during chunk allocation. Homogeneous power allocation and bit loading are then performed in a subsequent step to satisfy bit error rate (BER) constraint. The second one is the Round Robin (RR) which allocates chunks fairly among users regardless of their channel conditions. Both algorithms exploit the common concept of FFR for user differentiation during chunk allocation, but waste frequency resources on users under very poor channel conditions unlike our proposed algorithm. Although additional complexity is added by our proposed algorithm and signal-to-interference plus noise ratio (SINR) feedback is required at the transmitter ${ }^{2}$ compared to the two reference algorithms, the increase in system average spectral efficiency (SE) and fairness among users would tolerate this price.

\footnotetext{
${ }^{1}$ Authors assumed that this allocation methodology guarantees fairness

${ }^{2}$ Modern wireless system such as LTE-A already includes SINR feed-back
} 


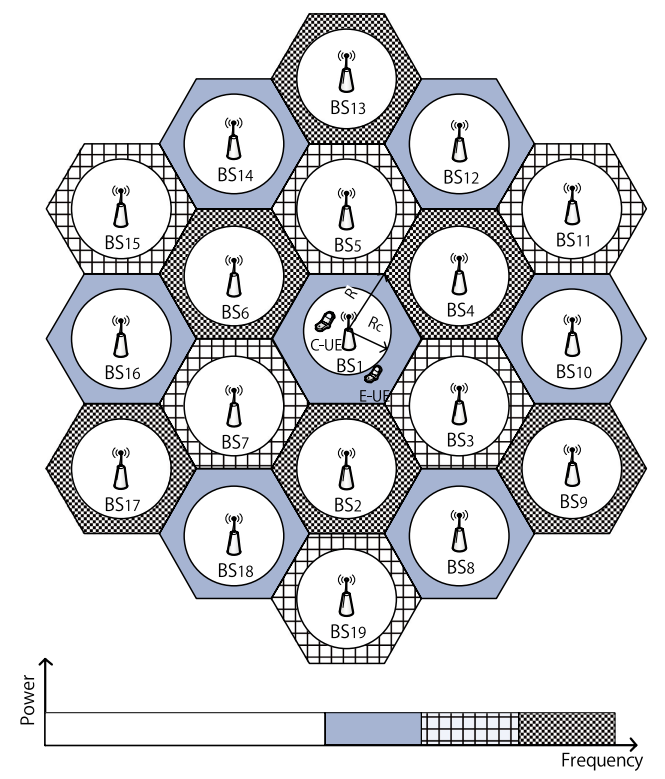

Fig. 1. Two-layer cellular system with FFR.

\section{System model}

Fig. 1 shows a two-layer multi-cell OFDMA downlink system model consisting of 19 macro-cells of radius $R$, each with single antenna macro base-station located at its center. Center-region of each macro-cell is bounded by the FFR radius, $R_{c}$, while edge-region is located elsewhere. Two different reuse factors of 1 and $1 / 3$ are used for center-region and edge-region, respectively. Total system bandwidth $B$ is divided equally into $N$ orthogonal narrowband flat fading sub-channels. $K$ active users, each with single receive antenna, are uniformly distributed within coverage area of the center macro-cell.

$M$ contiguous sub-channels are grouped into one chunk, denoted as $c, c \in$ $\{1,2, \ldots, C\}$, where $C=\left\lfloor\frac{N}{M}\right\rfloor$ is the number of chunks in the system where $\lfloor x\rfloor$ stands for the integer part of $x$. The normalized power frequency response channel vector associated with any user $k$ over any chunk $c, c=1,2, \ldots, C$ from $B S_{i}$ is $\mathbf{h}_{i, k, c}=\left[h_{i, k,(c-1) M+1}, \ldots, h_{i, k, c M}\right]$ and $h_{i, k, n}, n=(c-1) M+1, \ldots, c M$, is the frequency response of sub-channels belonging to chunk $c$. The corresponding channel magnitude, denoted as $\alpha_{i, k, c}$, is obtained by

$$
\alpha_{i, k, c}=\left(\frac{\mathbf{h}_{i, k, c} \mathbf{h}_{i, k, c}^{H}}{M}\right)^{\frac{1}{2}}
$$

where $(.)^{H}$ stands for conjugate transpose and $\alpha_{i, k, c}$ is independently and identically distributed $\forall i, k, c$ with unitary mean square, $E\left(\alpha_{i, k, c}^{2}\right)=1$. The total encountered loss between $B S_{i}$ and user $k$, denoted as $g_{i, k}$, is given by $g_{i, k}=d_{i, k}^{-\lambda} \cdot 10^{-X_{\sigma} / 10}$ where $d_{i, k}$ is the distance in-between, $X_{\sigma}$ is log-normal shadowing in (dB) and $\sigma$ is its standard deviation in $(\mathrm{dB})$.

Both center and edge region transmit powers are equally allocated among their corresponding chunks subject to the total transmit power constraint $P_{\max }$ based on analysis in [6]. Moreover, any sub-channel $n$ belonging to chunk $c$ associated with user $k$ is allocated the same power $p_{k, n}=P_{C}=P_{E}$ where $P_{C}$ and $P_{E}$ are the sub- 
channel power within center and edge areas, respectively. Chunk allocation is assumed to be globally known among all BSs. For any user $k$ located in the center cell, the average power received on any sub-channel $n$ within chunk $c$ is given by

$$
P_{r}= \begin{cases}P_{C} \cdot \alpha_{1, k, c}^{2} \cdot g_{i, k} & \text { if user } k \text { is center area user } \\ P_{E} \cdot \alpha_{1, k, c}^{2} \cdot g_{i, k} & \text { if user } k \text { is edge area user }\end{cases}
$$

The co-channel interference associated with any user $k$, denoted as $I_{k}$, is generated from the whole 18 macro BSs for users in center area while interference is generated from a specific set, $E=\{8,10,12,14,16,18\}$, in case of users in edge area. The variance of $I_{k}$, denoted as $\sigma_{I_{k}}^{2}$, is given by

$$
\sigma_{I_{k}}^{2}= \begin{cases}\sum_{i=2}^{19} P_{C} \cdot g_{i, k} & \text { if user } k \text { is center area user } \\ \sum_{i \in E} P_{E} \cdot g_{i, k} & \text { if user } k \text { is edge area user }\end{cases}
$$

Gaussian approximation of fading coefficient in (3) is validated by the assumption that number of interferers for both types of users is larger than one. Therefore, average SINR for any user $k$ over any chunk $c$, denoted as $\gamma_{k, c}$, is given by

$$
\gamma_{k, c}=\frac{P_{r}}{\sigma_{I_{k}}^{2}+\sigma_{\eta}^{2}}= \begin{cases}\frac{P_{C} \cdot \alpha_{1, k, c}^{2} \cdot g_{i, k}}{\sum_{i=2}^{19} P_{C} \cdot g_{i, k}+\sigma_{\eta}^{2}} & \text { if user } k \text { is center area user } \\ \frac{P_{E} \cdot \alpha_{1, k, c}^{2} \cdot g_{i, k}}{\sum_{i \in E} P_{E} \cdot g_{i, k}+\sigma_{\eta}^{2}} & \text { if user } k \text { is edge area user }\end{cases}
$$

where $\sigma_{\eta}^{2}$ is the variance of noise power.

We assume that all sub-channels within any chunk use the same bit loading and adaptive $l$-ary quadrature amplitude modulation (QAM) is exploited. For $l$-ary QAM modulation, the average BER encountered by any user $k$ on any sub-channel within chunk $c$ can be approximated as [7] $B E R_{k, c} \approx 0.2 \exp \left(\frac{m . \gamma_{k, c}}{l_{k, c}-1}\right)$ where $m=$ -1.6 and $l_{k, c}$ is the corresponding modulation level which is formulated, under a BER constraint $B E R_{t h}$, as

$$
l_{k, c}=\max _{l \in L}\left\{l \mid l \leq\left\lfloor 1+\frac{m \cdot \gamma_{k, c}}{\ln \left(5 B E R_{t h}\right)}\right\rfloor\right\}
$$

where $L$ is the set of available QAM modulation levels. Then, the data rate transmitted on each sub-channel belonging to chunk $c$ by any user $k, r_{k, c}$, is given by

$$
r_{k, c}= \begin{cases}\log _{2} l_{k, c} & \text { if user } k \text { is center area user } \\ \log _{2}\left(l_{k, c}\right) / 3 & \text { if user } k \text { is edge area user }\end{cases}
$$

The total rate achieved by any user $k, R_{k}$, is given by $R_{k}=\sum_{c=1}^{C} a_{k, c} \cdot M \cdot r_{k, c}$ where $a_{k, c} \in\{0,1\}$ is a binary parameter to determine chunk usage.

\section{Proposed chunk-based RA algorithm}

The proposed algorithm, described in details in Algorithm 1, is initialized by $R_{k}=0, \forall k \in \psi$ and $\rho_{c}=\emptyset, \forall c \in \Lambda$ where $\rho_{c}$ is the user selected for transmission over chunk $c$. The first step is a round robin step that prevents starvation for users with poor channel conditions such that, successively, each user $k \in \psi$ is allocated 


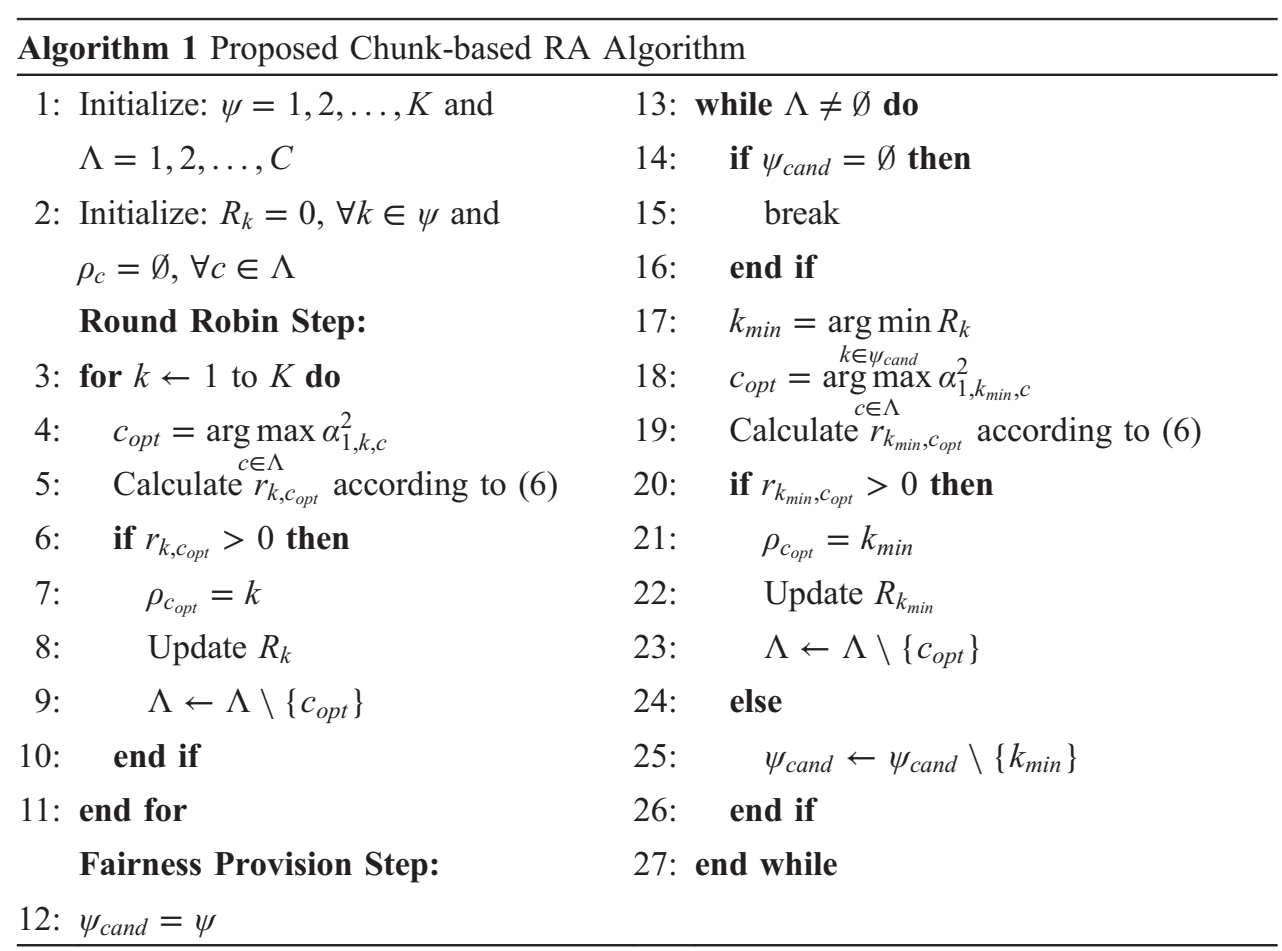

the chunk $c_{\text {opt }}$ with the highest channel magnitude among the set of chunks available in $\Lambda$. If the rate achieved on chunk $c_{\text {opt }}$ by user $k \in \psi$ is above zero, the chunk $c_{\text {opt }}$ is assigned to user $k$, removed from the set of chunks $\Lambda$ and the total rate, $R_{k}$, is updated. Otherwise, the loop continues for the next user.

A fairness provision step comes next to allocate the remaining chunks in $\Lambda$. A set of candidate users, $\psi_{\text {cand }}$, is initialized by $\psi_{\text {cand }}=\psi$ and the user $k_{\min }=$ $\underset{k \in \psi_{\text {cand }}}{\arg \min } R_{k}$ is selected to enhance fairness implicitly. The user $k_{\min }$ then selects the

chunk with the highest channel magnitude, denoted as $c_{\text {opt }}$, among the set $\Lambda$. If the rate achieved by user $k_{\min }$ on the chunk $c_{\text {opt }}$ is above zero, $c_{\text {opt }}$ is assigned to user $k_{\min }, R_{k_{\min }}$ is updated and $c_{\text {opt }}$ is removed from the set of chunks $\Lambda$. Otherwise, user $k_{\min }$ is removed from the set of candidate users $\psi_{\text {cand }}$ as it will not achieve rate over any other chunk as long as it does not achieve rate on its optimal chunk. The algorithm is terminated either if the set of candidate users $\psi_{\text {cand }}$ or the set of chunks $\Lambda$ become a null set. If the set of chunks $\Lambda$ is not empty at the algorithm termination, these chunks are considered in outage.

\section{Simulation \& results}

We compare our proposed algorithm in terms of average system SE and fairness among users to the reference algorithm in [5] and the RR algorithm. Inter-site distance is $500 \mathrm{~m}$, and bandwidth is $100 \mathrm{MHz}$ divided into 1024 sub-channels. Pathloss exponent $\lambda$ and shadowing standard deviation $\sigma$ are 3 and 8, respectively. BER constraint is set to $10^{-3}$. Users are uniformly distributed within the whole coverage area of the center cell and number of users, $K$, is set to 8 [5]. Average transmit signal to noise power ratio $S N R=\frac{P_{C}+P_{E}}{\sigma_{\eta}^{2}}=20 \mathrm{~dB}$, and number of subchannels per chunk, M, is set to 12 . 


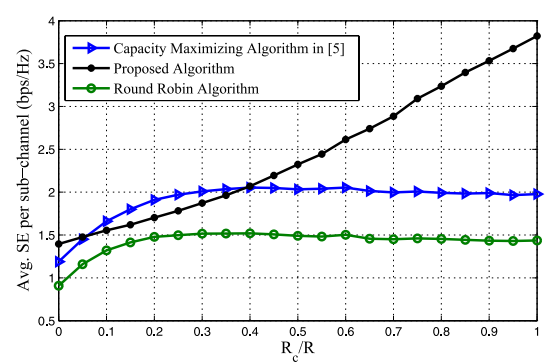

(a) Average Sum Rate vs. FFR radius ratio.

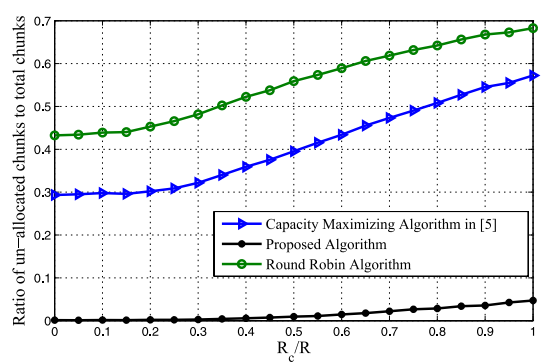

(b) Unallocated chunks ratio vs. FFR radius ratio.

Fig. 2. System SE performance of different algorithms.

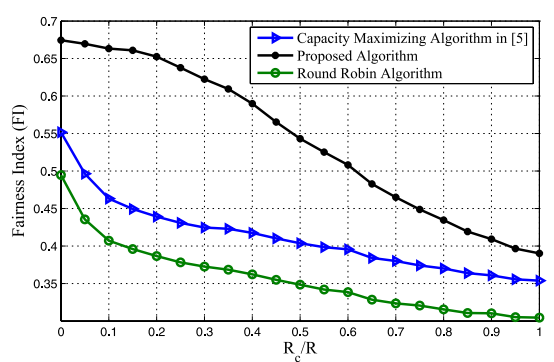

(a) Fairness index vs. FFR radius ratio.

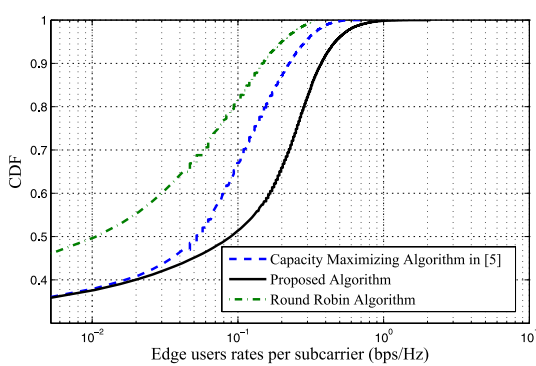

(b) CDF of edge users rates.

Fig. 3. Fairness performance of different algorithms.

Fig. 2(a) shows the average system SE per sub-channel against FFR radius ratio $R_{c} / R$ for the different algorithms. Results reveal that up to a specific radius ratio of 0.4 , the algorithm in [5] has better performance in terms of average SE due to low co-channel interference (CCI) generated from neighbouring cells. As FFR radius increases, center-region area increases and therefore users encounter strong CCI which limits the increase of achievable rates as more chunks are considered in outage as explained in Fig. 2(b). Fig. 2(b) shows the ratio of outage chunks at each algorithm termination to the total chunks against FFR radius ratio. For the algorithm in [5], as FFR radius increases, more users are subject to strong CCI and more chunks are in outage. In contrast, our proposed algorithm achieves lower outage as it avoids wasting chunks on users suffering from strong CCI. If any user fails to achieve rate on its optimal chunk, it is useless to include it furthermore in chunk competition as explained in Algorithm 1.

We exploit Jain's fairness index (FI) [8], defined as $F I=\frac{\left(\sum_{k} R_{k}\right)^{2}}{K \cdot \sum_{k} R_{k}^{2}}$ to measure fairness. Fig. 3(a) shows the FI of different algorithms against FFR radius ratio. Our proposed algorithm highly increases fairness compared to the two reference algorithms by considering the user with minimum rate during every chunk allocation. Our proposed algorithm provides $50 \%$ fairness gain compared to the algorithm in [5] for the same SE. For further comparison, cumulative distribution function (CDF) of rates per sub-channel associated with cell-edge users (i.e., users in edge-region) are shown in Fig. 3(b) at FFR radius ratio of 0.4 for fair comparison purpose. Our fairness-aware RA algorithm has clearly enhanced celledge users performance by considering users with the minimum rate. 


\section{Conclusion}

We have proposed a fairness-aware chunk-based RA algorithm for the downlink transmission of multi-user multi-cell OFDMA systems adopting FFR. Simulation results reveal that our proposed algorithm exceeds the two reference algorithms in terms of average system SE by avoiding wasting resources on users with poor channel conditions. Fairness among cell-edge users and other users rates has been also enhanced by considering users with the minimum rate during every chunk allocation. 